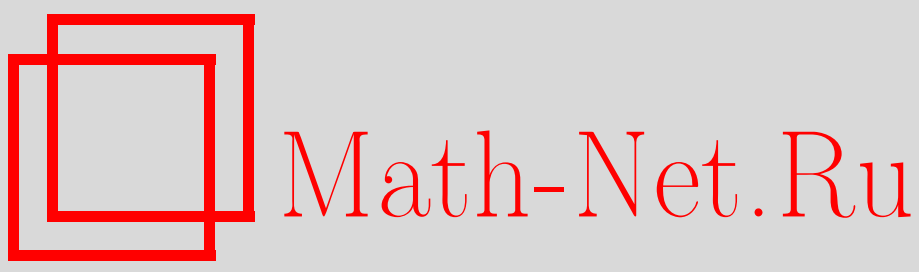

К. Н. Панков, Асимптотические оценки для чисел двоичных отображений с заданными криптографическими свойствами, Матем. вопр. криптогр., 2014, том 5, выпуск 4, 73-97

DOI: https://doi.org/10.4213/mvk136

Использование Общероссийского математического портала Math-Net.Ru подразумевает, что вы прочитали и согласны с пользовательским соглашением

http://www. mathnet.ru/rus/agreement

Параметры загрузки:

IP : 54.80 .97 .219

26 апреля 2023 г., 05:26:28

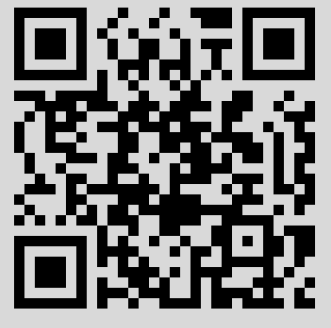


МАТЕМАТИЧЕСКИЕ ВОПРОСЫ КРИПТОГРАФИИ

2014 Т. 5 № 4 C. $73-97$

УДК $519.212 .2+519.214$

\title{
Асимптотические оценки для чисел двоичных отображений с заданными криптографическими свойствами
}

\author{
К. Н. Панков
}

Московский государственный технический университет радиотехники, электроники и автоматики, г. Москва

Получено 22.IV.2013

Уточнена локальная предельная теорема для распределения части вектора весов подфункций и доказана локальная предельная теорема для распределения части вектора спектральных коэффициентов линейных комбинаций координатных функций случайного двоичного отображения. Найдены верхние и нижние асимптотические оценки для числа корреляционно-иммунных и $k$-эластичных двоичных вектор-функций, а также верхняя оценка для числа платовидных отображений.

Ключевые слова: случайное двоичное отображение, локальная предельная теорема, спектральные коэффициенты, корреляционноиммунные вектор-функции, устойчивые вектор-функции, платовидные вектор-функции.

\section{Asymptotic estimates for numbers of Boolean mappings with given cryptographic properties \\ K. N. Pankov \\ Moscow State Institute of Radio Engineering, Electronics and Automation, Moscow}

Abstract. For linear combinations of coordinate functions of random Boolean mapping a local limit theorem for the distribution of subsets of weights of submappings is improved. Also a local limit theorem for subsets of their spectral coefficients is proved. By means of these theorems we obtain upper and lower asymptotic estimates for numbers of correlation-immune and $(n, m, k)$-resilient Boolean mappings. Also we obtain an upper asymptotic estimate of the number of plateaued Boolean mappings.

Keywords: random binary mapping, local limit theorem, spectral coefficient, correlation-immune Boolean mapping, resilient Boolean mapping, plateaued Boolean mapping.

Citation: Mathematical Aspects of Cryptography, 2014, vol. 5, no. 4, pp. 73-97 (Russian)

(C) 2014 K. Н. Панков 


\section{Введение}

Некоторые свойства двоичных отображений и определяемые ими классы вектор-функций, как отмечено в монографии [6], выделялись в связи с реальными задачами анализа и синтеза шифров. $\mathrm{K}$ таким классам относятся корреляционно-иммунные, устойчивые и, отчасти, платовидные отображения. Например, устойчивые вектор-функции используются в поточных шифрах в качестве комбинирующих функций, обладающих способностью противостоять корреляционному методу криптоанализа, так как их выход статистически не зависит от некоторых комбинаций входов. Согласно [6, с. 308] корреляционно-иммунные функции связаны с простыми ортогональными массивами (таблицами), изучающимися в комбинаторике и статистике при планировании экспериментов, а в криптографии - при построении кодов аутентификации [3, с. 148]. Эти функций связаны также с линейными кодами с (некоторым) дуальным расстоянием, изучающимися в теории кодирования [11, с. 143]. Класс платовидных функций является обобщением класса бент-функций, устойчивых, например, к линейному методу криптографического анализа.

Пусть $V_{n}$ - множество двоичных векторов размерности $n,|A|$ - мощность множества $A, \overline{1, k}=\{1, \ldots, k\}, B_{n}^{m}=\left\{f \mid f: V_{n} \rightarrow V_{m}\right\}$ - множество отображений из $V_{n}$ в $V_{m}$, для $f\left(x_{1}, \ldots, x_{n}\right) \in B_{n}^{m}$ будем рассматривать подфункции $f_{i_{1}, \ldots, i_{k}}^{a_{1}, \ldots, a_{k}}=\left(\left(f_{1}\right)_{i_{1}, \ldots, i_{k}}^{a_{1}, \ldots, a_{k}}, \ldots\left(f_{m}\right)_{i_{1}, \ldots, i_{k}}^{a_{1}, \ldots, a_{k}}\right) \in B_{n-k}^{m}$, где $\left(f_{j}\right)_{i_{1}, \ldots, i_{k}}^{a_{1}, \ldots, a_{k}}$ подфункция булевой функции $f_{j} \in B_{n}^{1}$, получающаяся, если у аргумента $f_{j}$ значения координат с номерами $i_{1}, \ldots, i_{k}$ положить равными $a_{1}, \ldots, a_{k}$. Отображение $f\left(x_{1}, \ldots, x_{n}\right) \in B_{n}^{m}$ называется корреляиионно-иммунным порядка $k$, если для любых наборов $1 \leq i_{1}<\ldots<i_{k} \leq n$, для любых $a_{j} \in\{0,1\}, j \in \overline{1, k}$, выполняется условие

$$
\left|\left\{\bar{u} \in V_{n-k}: f_{i_{1}, \ldots, i_{k}}^{a_{1}, \ldots, a_{k}}(\bar{u})=\bar{v}\right\}\right|=2^{-k}\left|\left\{\bar{w} \in V_{n}: f(\bar{w})=\bar{v}\right\}\right|
$$

для всех $\bar{v} \in V_{m}$.

Корреляционно-иммунное порядка $k$ отображение $f\left(x_{1}, \ldots, x_{n}\right) \in B_{n}^{m}$ называется $(n, m, k)$-устойчивым, если для любого вектора $\bar{v} \in V_{m}$ верно равенство

$$
\left|\left\{\bar{w} \in V_{n}: f(\bar{w})=\bar{v}\right\}\right|=2^{n-m}
$$

В “Словаре криптографических терминов" вводятся термины “функция корреляционно-иммунная порядка $k$ " [10, с. 78] в одномерном случае и "отображение $k$-корреляционно-иммунное" [10, с. 78] в многомерном. Также вводится термин “функция $k$-эластичная" [10, с. 81], эквивалент- 
ный термину “( $n, 1, k)$-устойчивое отображение”. В соответствии с логикой "Словаря" в многомерном случае можно вместо термина “ $(n, m, k)$ устойчивые отображения" использовать "отображсния $k$-эластичные".

В западной литературе $k$-эластичные функции рассматривались в известной работе Т. Зигенталера [21], и при $m=1$ назывались $k$ устойчивыми. В СССР аналогичным образом введенные булевы функции изучались в 70-х годах Л. В. Ларионовым.

Изучению свойств корреляционно-иммунных функций в случае $m=1$ посвящено множество работ, из которых можно отметить $[5,12,11,6]$, а изучению свойств $k$-эластичных функций, введенных независимо в работах $[19,15]$, в случае $m>1$ были посвящены работы $[20,17,22,23]$. В работах $[1,2,7]$ были найдены асимптотические оценки для мощности множества $k$-эластичных функций при различных значениях $k$ и $m \in \overline{1,4}$.

В монографии [6, с. 310] было доказано, что существование $k$ эластичного отображения эквивалентно существованию покрывающего множества из некоторого количества ортогональных массивов определенного вида.

Согласно [16] $k$-эластичные отображения связаны с такими областями, как выработка общих ключей для квантово-криптографических каналов и построение генераторов псевдослучайных последовательностей для поточных шифров.

В [13] было доказано, что многие важные свойства двоичного отображения $f$ имеют место, если этими свойствами обладают все ненулевые линейные комбинации координатных функций $f$, называемых в [16] компонентными функциями или компонентами. Свойства же компонент можно, в частности, выразить в терминах их коэффициентов Фурье-Уолша-Адамара (спектральных коэффициентов) и весов подфункций.

Далее, как и в работе [7], под обозначением $\psi_{m}(J)$, где $J=\left\{j_{1}, \ldots, j_{|J|}\right\}$ - подмножество множества $\overline{1, m}$, мы будем понимать двоичный вектор из $V_{m}$, у которого координаты с номерами $j_{1}, \ldots, j_{|J|}$ равны единице, а остальные - нулю, а под $(\bar{x}, \bar{y})=x_{1} y_{1} \oplus \ldots \oplus x_{n} y_{n}$ - скалярное произведение векторов $\bar{x}$ и $\bar{y}$ из $V_{m}$. В терминологии [9] $\psi_{m}(J)$ называется индикаторным вектором множества $J$.

Для произвольных подмножества $I=\left\{i_{1}, \ldots, i_{|I|}\right\}$ множества $\overline{1, n}$ и непустого подмножества $J=\left\{j_{1}, \ldots, j_{|J|}\right\}$ множества $\overline{1, m}$ через $w_{I}^{J}(f)$ обозначим вес $\left\|\left(\psi_{m}(J), f\right)_{i_{1}, \ldots, i_{|I|}}^{1, \ldots, 1}\right\|$ подфункции $\left(\psi_{m}(J), f\right)_{i_{1}, \ldots, i_{|I|}}^{1, \ldots, 1}$ компоненты $\left(\psi_{m}(J), f\right)=f_{j_{1}} \oplus \ldots \oplus f_{j_{|J|}}$ отображения $f=\left(f_{1}, \ldots, f_{n}\right) \in B_{n}^{m}$, получаемой, если у аргумента компоненты $\left(\psi_{m}(J), f\right)$ значения координат с номерами $i_{1}, \ldots, i_{|I|}$ положить равными единице.

В таких же условиях для компоненты $\left(\psi_{m}(J), f\right)$ можно определить 
спектральный коэффициент Фурье-Уолша-Адамара [8]

$$
\begin{aligned}
\Delta_{I}^{J}(f)=\frac{1}{2} \sum_{x \in V_{n}}(-1)^{\left(\psi_{m}(J), f\right)(x) \oplus x_{i_{1}} \oplus \ldots \oplus x_{i_{|I|}}}= \\
=2^{n-1}-\left\|\left(\psi_{m}(J), f\right)(x) \oplus\left(\psi_{n}(I), x\right)\right\|,
\end{aligned}
$$

где $\left(\psi_{n}(I), x\right)=x_{i_{1}} \oplus \ldots \oplus x_{i_{|I|}} ;$ согласно [10, с. 71] называется коэффиииентом статистической структуры компоненты $\left(\psi_{m}(J), f\right)$.

Как уже упоминалось в работе [7], многие свойства двоичных отображений зависят от того, чему равен вектор, состоящий из определенных выше характеристик всех компонент или их части. В частности, двоичное отображение является корреляционно-иммунным порядка $k$, если набор

$$
\left(w_{I}^{J}(f)-2^{n-|I|-1}: \forall J \subset \overline{1, m}, J \neq \varnothing, \forall I \subset \overline{1, n}, 1 \leq|I| \leq k\right)
$$

или набор

$$
\left(\Delta_{I}^{J}(f): \forall J \subset \overline{1, m}, J \neq \varnothing, \forall I \subset \overline{1, n}, 1 \leq|I| \leq k\right)
$$

состоят из одних нулей $[2,13]$.

Согласно [6, с. 272] функция из $B_{n}^{1}$ называется платовидной функиией порядка $2 r$, если квадрат каждого коэффициента ее статистической структуры равен либо $2^{2 n-2 r-2}$, либо 0 .

Изучению класса платовидных функций посвящен ряд работ, из которых можно отметить работы $[4,6,16]$. Из равенства Парсеваля следует, что число ненулевых элементов среди всех коэффициентов этих функций равно $2^{2 r}$ и что $0 \leq r<n / 2$.

Согласно [16, с. 418] двоичное отображение $f(x) \in B_{n}^{m}$ называется платовидным, если платовидны все его компоненты.

Представляется естественным ввести понятие порядка для платовидного отображения. Порядком платовидного отображсения будем называть вектор $\left(r_{J}, \varnothing \neq J \subset \overline{1, m}\right)$, составленный из порядков $2 r_{J}$ всех его компонент $\left(\psi_{m}(J), f\right)$, взятых в лексикографическом порядке. В [16, c. 418] величины $r_{J}$ называются амплитудами (amplitude) платовидных компонент $\left(\psi_{m}(J), f\right)$.

Пусть функция $f$ выбирается случайно и равновероятно из множества $B_{n}^{m}$. Это эквивалентно независимому равновероятному выбору ее значений $f(\alpha)$ из множества $V_{m}$ для всех $\alpha$ из множества $V_{n}$. Можно рассматривать значение функции $f$ как вектор размерности $m$, состоящий из значений ее координатных двоичных функций от $n$ переменных:

$$
f(\alpha)=\left(f_{1}(\alpha), f_{2}(\alpha), \ldots, f_{m}(\alpha)\right): V_{n} \rightarrow V_{m},
$$


где $f_{i}(\alpha): V_{n} \rightarrow\{0,1\}$ для всех $i \in \overline{1, m}$.

Тогда значения $f_{i}(\alpha)$ выбираются независимо и равновероятно из множества $\{0,1\}$ для всех $\alpha$ из множества $V_{n}$ и для всех $i \in \overline{1, m}$.

При случайном выборе $f$ векторы, состоящие из части характеристик компонент отображения, также являются случайными, и возникает вопрос об их распределении. В [8] была доказана асимптотическая нормальность векторов фиксированной размерности, состоящих из коэффициентов статистической структуры и весов подфункций компонент случайной двоичной вектор-функции, с оценкой скорости сходимости, а в [7] - локальная предельная теорема для распределения части вектора весов подфункций двоичного отображения растущей размерности, обобщающая на случай широкого класса значений $m$ результат из [2].

В первом разделе данной работы уточнена локальная предельная теорема для распределения части вектора весов подфункций компонент случайного двоичного отображения при $m=2,3$ и найдены асимптотические верхние и нижние оценки для мощности класса $k$-эластичных двоичных отображений. Во втором разделе получена асимптотическая верхняя оценка для мощности класса корреляционно-иммунных функций. В третьем разделе доказана локальная предельная теорема для распределения части вектора коэффициентов статистической структуры двоичного отображения растущей размерности и найдена асимптотическая верхняя оценка для для мощности класса платовидных отображений при заданных ограничениях на его порядок.

\section{1. Верхние и нижние асимптотические оценки для числа $k$ - эластичных двоичных отображений}

Определим случайный вектор

$$
\bar{w}=\bar{w}(n, m, k)=\left(w^{J}, \varnothing \neq J \subset \overline{1, m}\right),
$$

где $w^{J}=\left(w_{I}^{J}(f), I \subset \overline{1, n}, 0 \leq|I| \leq k\right)$, размерности

$$
N(n, m, k)=\left(2^{m}-1\right) L(n, k), \text { где } L(n, k)=\sum_{i=0}^{k}\left(\begin{array}{c}
n \\
i
\end{array}\right) .
$$

Согласно следствию 1 [8] веса подфункций двоичного отображения удовлетворяют сравнению

$$
\sum_{\varnothing \neq S \subset J}(-1)^{|S|} w_{I}^{S} \equiv 0\left(\bmod 2^{|J|-1}\right) .
$$


Здесь и далее будем использовать обозначения $N=N(n, m, k)$, $\exp _{2} x=2^{x}$.

В [7] была доказана следующая теорема.

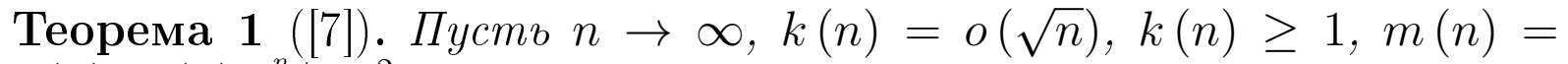
о $(n), \bar{z}(n) 2^{\frac{n}{2}+m-2}-$ такая последовательность иелочисленных векторстолбцов размерности $N(n, m, k)$, что координаты векторов из последовательности $\mathrm{E} \bar{w}+\bar{z} 2^{\frac{n}{2}+m-2}$ удовлетворяют сравнениям (1). Тогда равномерно относительно $\bar{z}=\bar{z}(n)$ верно равенство

$$
\begin{aligned}
& \mathrm{P}\left(\bar{w}=\mathrm{E} \bar{w}+\bar{z} 2^{\frac{n}{2}+m-2}\right)=\left(\sum_{\vec{r} \in \Re^{* *}(m, N)} \exp \left[-i \pi\left(2^{\frac{n}{2}} \vec{r}, \bar{z}\right)\right]\right) \times \\
& \times \frac{\exp \left(-2^{2 m-3} \sum_{\varnothing \neq J \subset \overline{1, m}} \sum_{\substack{c \subset \overline{1, n},|I| \leq k}}\left(\sum_{K \subset I}(-1)^{|K|} 2^{|K|} z_{K}^{J}\right)^{2}\right)+O\left(n^{3 k+3} 2^{3 m-\frac{n}{2}}\right)}{\exp _{2}\left(\frac{(n-k)}{2}\left(\begin{array}{l}
n \\
k
\end{array}\right)\left(2^{m}-1\right)+N(n, m, k) \log _{2} \sqrt{\frac{\pi}{2}}\right)},
\end{aligned}
$$

где $\bar{z}$ имеет такую же структуру координат как $\bar{w}$

$$
\begin{gathered}
\mathfrak{R}^{* *}(m, N)=\left\{\vec{r} \in\left\{0, \ldots, 2^{m-1}-1\right\}^{N}: \forall I, \forall s \in \overline{1, m}, \forall \delta \in V_{m}\right. \\
\left.\sum_{J \subset \overline{1, m}: s \in J}(-1)^{\left(\delta, \psi_{m}(J)\right) \oplus 1} r_{I}^{J} \equiv 0\left(\bmod 2^{m-1}\right)\right\} .
\end{gathered}
$$

Для упрощения записи полученных далее результатов введем обозначение

$$
T(n, m, k)=\frac{n-k}{2}\left(\begin{array}{l}
n \\
k
\end{array}\right)\left(2^{m}-1\right)+N(n, m, k) \log _{2} \sqrt{\frac{\pi}{2}} .
$$

Множество функций, являющихся $k$-эластичными, обозначим через $R[n, m, k]$. Любая ненулевая комбинация координатных функций этих функций является уравновешенной корреляционно-иммунной функцией.

Также в [7] была доказана асимптотическая формула для мощности множества $R[n, m, k]$ при $n \rightarrow \infty, k(n)=o(\sqrt{n}), m(n)=o(n)$ :

$$
|R[n, m, k]| \sim\left|\mathfrak{R}^{* *}(m, N)\right| \exp _{2}\left(m 2^{n}-T(n, m, k)\right),
$$

где множество $\mathfrak{R}^{* *}(m, N)$ определено выше (см. (2)).

Докажем вспомогательное утверждение. 
Утверждение 1. Если $\vec{r} \in \mathfrak{R}^{* *}=\mathfrak{R}^{* *}(m, N)$, то для любого $I \subset \overline{1, n}$, $|I| \leq k$ в векторе $\left(r_{I}^{J}, \varnothing \neq J \subset \overline{1, m}\right)$ все координаты имеют одинаковую четность.

Доказательство. Докажем данное утверждение индукцией по $m$.

Пусть $m=2$. Тогда $r_{I}^{J} \in\{0,1\}$, а множество $\mathfrak{R}^{* *}$ имеет вид

$$
\begin{aligned}
\mathfrak{R}^{* *}=\left\{\vec{r} \in\{0,1\}^{N(n, 2, k)}: \forall I, \forall s \in \overline{1,2}, \forall \delta \in V_{2}\right. \\
\left.\qquad \sum_{J \subset \overline{1,2}: s \in J}(-1)^{\left(\delta, \psi_{2}(J)\right) \oplus 1} r_{I}^{J} \equiv 0(\bmod 2)\right\} .
\end{aligned}
$$

Рассмотрим систему сравнений

$$
\sum_{J \subset \overline{1,2}: s \in J}(-1)^{\left(\delta, \psi_{2}(J)\right) \oplus 1} r_{I}^{J} \equiv 0(\bmod 2): \forall I \subset \overline{1, n},|I| \leq k, \forall s \in \overline{1,2}, \forall \delta \in V_{2} .
$$

Зафиксируем $I \subset \overline{1, n},|I| \leq k$. Тогда одновременно выполняются две системы сравнений

$$
\left\{\begin{array} { c } 
{ r _ { I } ^ { \{ 1 \} } + r _ { I } ^ { \{ 1 , 2 \} } \equiv 0 ( \operatorname { m o d } 2 ) , } \\
{ r _ { I } ^ { \{ 1 \} } - r _ { I } ^ { \{ 1 , 2 \} } \equiv 0 ( \operatorname { m o d } 2 ) , }
\end{array} \text { и } \quad \left\{\begin{array}{l}
r_{I}^{\{2\}}+r_{I}^{\{1,2\}} \equiv 0(\bmod 2) \\
r_{I}^{\{2\}}-r_{I}^{\{1,2\}} \equiv 0(\bmod 2) .
\end{array}\right.\right.
$$

Решением ее являются векторы $\left(r_{I}^{\{1\}}, r_{I}^{\{2\}}, r_{I}^{\{1,2\}}\right) \in\{(0,0,0),(1,1,1)\}$ (а также любые векторы, координаты которых имеют одинаковую четность).

Итак, пусть предположение верно при $m=t$.

Тогда решением системы сравнений

$$
\sum_{J \subset \overline{1, t} \backslash s\}}(-1)^{\left(\delta, \psi_{t-1}(J)\right)} r_{I}^{J \cup\{s\}} \equiv 0\left(\bmod 2^{t-1}\right) \quad \forall s \in \overline{1, t}, \quad \forall \delta \in V_{t-1},
$$

является вектор, все координаты которого имеют одинаковую четность.

Рассмотрим случай, когда $m=t+1$.

Так как $\vec{r} \in \mathfrak{R}^{* *}$, то $\sum_{J \subset 1, t+1: s \in J}(-1)^{\left(\delta, \psi_{t+1}(J)\right) \oplus 1} r_{I}^{J} \equiv 0\left(\bmod 2^{t}\right)$ при любых $s \in \overline{1, t+1}, \delta \in V_{t+1}$. Очевидно, что эта система сравнений эквивалентна системе

$$
\sum_{J \subset \overline{1, t+1} \backslash\{s\}}(-1)^{\left(\delta, \psi_{t}(J)\right)} r_{I}^{J \cup\{s\}} \equiv 0\left(\bmod 2^{t}\right) \forall s \in \overline{1, t+1}, \forall \delta \in V_{t} .
$$


Пусть $s=1, \delta=\left(\delta^{\prime}, \delta_{t}\right)$, где $\delta^{\prime} \in V_{t-1}, \delta_{t} \in\{0,1\} ;$ тогда

$$
\begin{aligned}
\sum_{J \subset \overline{1, t+1} \backslash\{1\}}(-1)^{\left(\delta, \psi_{t}(J)\right)} r_{I}^{J \cup\{1\}}=\sum_{J^{\prime} \subset \overline{1, t} \backslash\{s\}}(-1)^{\left(\delta, \psi_{t-1}\left(J^{\prime}\right)\right)} r_{I}^{J^{\prime} \cup\{1\}}+ \\
+(-1)^{\delta_{t}} \sum_{J^{\prime} \subset \overline{1, t} \backslash\{s\}}(-1)^{\left(\delta, \psi_{t-1}\left(J^{\prime}\right)\right)} r_{I}^{J^{\prime} \cup\{1\} \cup\{t+1\}} \equiv 0\left(\bmod 2^{t}\right) .
\end{aligned}
$$

Рассмотрев случаи, когда $\delta_{t}=0$ и $\delta_{t}=1$, получаем, что $\sum_{J^{\prime} \subset \overline{1, t} \backslash\{s\}}(-1)^{\left(\delta, \psi_{t-1}\left(J^{\prime}\right)\right)} r_{I}^{J \cup\{1\}}$ и $\sum_{J^{\prime} \subset \overline{1, t} \backslash\{s\}}(-1)^{\left(\delta, \psi_{t-1}\left(J^{\prime}\right)\right)} r_{I}^{J \cup\{1\} \cup\{t+1\}}$ сравнимы с нулем по модулю $2^{t-1}$ для любого $\delta^{\prime} \in V_{t-1}$.

Аналогично рассматривается случай, когда $s \in \overline{2, t}$.

Получаем две системы сравнений

$$
\begin{gathered}
\sum_{J \subset \overline{1, t} \backslash\{s\}}(-1)^{\left(\delta, \psi_{t-1}(J)\right)} r_{I}^{J \cup\{s\}} \equiv 0\left(\bmod 2^{t-1}\right): \forall s \in \overline{1, t}, \forall \delta \in V_{t-1}, \\
\sum_{J \subset \overline{1, t} \backslash\{s\}}(-1)^{\left(\delta, \psi_{t-1}(J)\right)} r_{I}^{J \cup\{s\} \cup\{t+1\}} \equiv 0\left(\bmod 2^{t-1}\right): \forall s \in \overline{1, t}, \forall \delta \in V_{t-1},
\end{gathered}
$$

в решениях которых согласно предположению индукции все координаты имеют одинаковую четность.

Если вместо $J^{\prime} \subset \overline{1, t} \backslash\{s\}$ суммировать по $J^{\prime} \subset \overline{1, t+1} \backslash\{s, 2\}$ и рассмотреть все $s \in \overline{1, t+1} \backslash\{2\}$, то мы получим еще две системы сравнений

$$
\begin{gathered}
\sum_{J^{\prime} \subset \overline{1, t+1} \backslash\{s, 2\}}(-1)^{\left(\delta, \psi_{t-1}(J)\right)} r_{I}^{J \cup\{s\}} \equiv 0\left(\bmod 2^{t-1}\right): \forall \delta \in V_{t-1}, \\
\sum_{J^{\prime} \subset \overline{1, t+1} \backslash\{s, 2\}}(-1)^{\left(\delta, \psi_{t-1}(J)\right)} r_{I}^{J \cup\{s\} \cup\{2\}} \equiv 0\left(\bmod 2^{t-1}\right): \forall \delta \in V_{t-1},
\end{gathered}
$$

в решениях которых согласно предположению индукции также все координаты имеют одинаковую четность. Так как векторы, являющиеся решениями этих систем - следствий исходной системы, попарно пересекаются и содержат все элементы вектора $\left(r_{I}^{J}, \varnothing \neq J \subset \overline{1, m},|J| \leq k-|I|\right)$, то обоснование шага индукции завершено.

С помощью доказанного утверждения можно уточнить формулировку теоремы [7], если $m \in\{2,3\}$. 
Утверждение 2. В условиях теоремы 1 [7], если $m \in\{2,3\}$, то

$$
\begin{aligned}
& \mathrm{P}\left(\bar{w}=\mathrm{E} \bar{w}+\bar{z} 2^{\frac{n}{2}+m-2}\right)=\frac{\exp \left(-\frac{1}{2} \bar{z}^{T} Q^{-1} \bar{z}\right)+O\left(n^{3 k-3} 2^{-\frac{n}{2}+3 m}\right)}{2^{\frac{n+2 m-3}{2} N} 2 \sqrt{\pi^{N} \operatorname{det} Q}}\left|\Re^{* *}\right|= \\
& =\frac{\exp \left(-2^{2 m-3} \sum_{\varnothing \neq J \subset \overline{1, m}} \sum_{\substack{c \subset 1, n \\
|I| \leq k}}\left(\sum_{K \subset I}(-1)^{|K|} 2^{|K|} z_{K}^{J}\right)^{2}\right)+O\left(n^{3 k+3} 2^{3 m-\frac{n}{2}}\right)}{\exp _{2}(T(n, m, k)-N(n, 1, k)(4 m-7))},
\end{aligned}
$$

где $Q=Q(n, m, k)$ - ковариачионнал матрица вектора $(\bar{w}-\mathrm{E} \bar{w}) 2^{-\frac{n}{2}-m+2}$.

Доказательство. При $m=2$ из утверждения 1 следует, что $\left(r_{I}^{\{1\}}, r_{I}^{\{2\}}, r_{I}^{\{1,2\}}\right) \in\{(0,0,0),(1,1,1)\}$. Тогда $\left|\mathfrak{R}^{* *}\right|=\exp _{2}((n, 1, k))$.

Теперь рассмотрим сумму

$$
\begin{aligned}
& \sum_{\vec{r} \in \Re^{* *}} \exp \left[-i \pi\left(2^{\frac{n}{2}} \vec{r}, \bar{z}\right)\right]= \\
& =\sum_{\vec{r} \in \Re^{* *}} \exp \left[-i \pi 2^{2-m}\left(\sum_{I \subset \overline{1, n}, I I \leq k} \sum_{\varnothing \neq J \subset \overline{1, m}} r_{I}^{J} \cdot z_{I}^{J} 2^{\frac{n}{2}+m-2}\right)\right]= \\
& =\sum_{\vec{r} \in \mathfrak{R}^{* *}} \exp \left[-i \pi\left(\sum_{I \subset \overline{1, n},|I| \leq k} \sum_{\varnothing \neq J \subset \overline{1,2}} r_{I}^{J} \cdot\left(\mathrm{E} w_{I}^{J}+z_{I}^{J} 2^{\frac{n}{2}}\right)\right)\right]=
\end{aligned}
$$

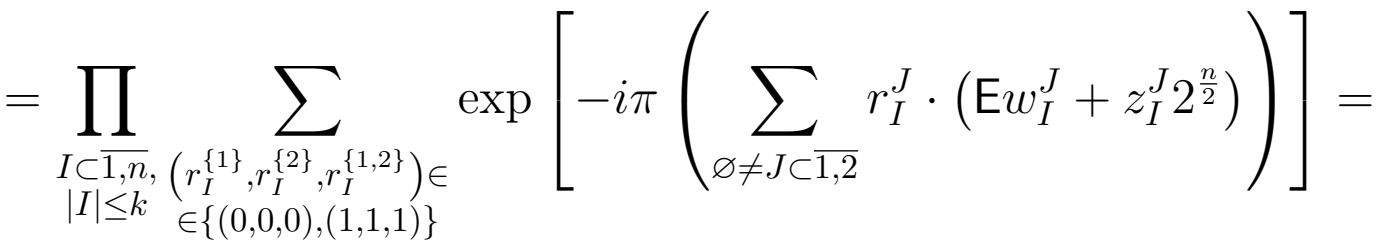

$$
\begin{aligned}
& =\prod_{\substack{I \subset 1, n,|I| \leq k}}\left(1+\exp \left[-i \pi\left(\sum_{\varnothing \neq J \subset \overline{1,2}}\left(\mathrm{E} w_{I}^{J}+z_{I}^{J} 2^{\frac{n}{2}}\right)\right)\right]\right) .
\end{aligned}
$$

Обозначим

$$
v_{I}^{J}=\mathrm{E} w_{I}^{J}+z_{I}^{J} 2^{\frac{n}{2}+m-2}=\mathrm{E} w_{I}^{J}+z_{I}^{J} 2^{\frac{n}{2}} \in \mathbb{Z} .
$$


Тогда из того, что $v_{I}^{J}$ удовлетворяют сравнениям (1) из следствия 1 [8], следует, что

$$
\begin{aligned}
\sum_{\varnothing \neq J \subset \overline{1,2}}\left(\mathrm{E} w_{I}^{J}+2^{n / 2}\right) & =v_{I}^{\{1,2\}}+v_{I}^{\{1\}}+v_{I}^{\{2\}}= \\
& =2\left(2^{-1} \sum_{\varnothing \neq J \subset \overline{1,2}}(-1)^{|J|} v_{I}^{J}\right)+2 v_{I}^{\{1\}}+2 v_{I}^{\{2\}} \in 2 \mathbb{Z} .
\end{aligned}
$$

Значит, $\sum_{\vec{r} \in \mathfrak{R}^{* *}} \exp \left[-i\left(\pi 2^{n / 2} \vec{r}, \bar{z}\right)\right]=\exp _{2}(L(n, k))=\left|\mathfrak{R}^{* *}\right|$.

Теперь пусть $m=3$. Зафиксируем $I \subset \overline{1, n},|I| \leq k$. Тогда аналогично доказательству утверждения 1 имеем систему из трех систем сравнений

$$
\begin{gathered}
\left\{\begin{array}{l}
r_{I}^{\{1\}}+r_{I}^{\{1,2\}}+r_{I}^{\{1,3\}}+r_{I}^{\{1,2,3\}} \equiv 0(\bmod 4), \\
r_{I}^{\{1\}}-r_{I}^{\{1,2\}}+r_{I}^{\{1,3\}}-r_{I}^{\{1,2,3\}} \equiv 0(\bmod 4), \\
r_{I}^{\{1\}}+r_{I}^{\{1,2\}}-r_{I}^{\{1,3\}}-r_{I}^{\{1,2,3\}} \equiv 0(\bmod 4), \\
r_{I}^{\{1\}}-r_{I}^{\{1,2\}}-r_{I}^{\{1,3\}}+r_{I}^{\{1,2,3\}} \equiv 0(\bmod 4),
\end{array}\right. \\
\left\{\begin{array}{l}
r_{I}^{\{2\}}+r_{I}^{\{1,2\}}+r_{I}^{\{2,3\}}+r_{I}^{\{1,2,3\}} \equiv 0(\bmod 4), \\
r_{I}^{\{2\}}-r_{I}^{\{1,2\}}+r_{I}^{\{2,3\}}-r_{I}^{\{1,2,3\}} \equiv 0(\bmod 4), \\
r_{I}^{\{2\}}+r_{I}^{\{1,2\}}-r_{I}^{\{2,3\}}-r_{I}^{\{1,2,3\}} \equiv 0(\bmod 4), \\
r_{I}^{\{2\}}-r_{I}^{\{1,2\}}-r_{I}^{\{1,3\}}+r_{I}^{\{1,2,3\}} \equiv 0(\bmod 4),
\end{array}\right. \\
\left\{\begin{array}{l}
r_{I}^{\{3\}}+r_{I}^{\{1,3\}}+r_{I}^{\{2,3\}}+r_{I}^{\{1,2,3\}} \equiv 0(\bmod 4), \\
r_{I}^{\{3\}}-r_{I}^{\{1,3\}}+r_{I}^{\{2,3\}}-r_{I}^{\{1,2,3\}} \equiv 0(\bmod 4), \\
r_{I}^{\{3\}}+r_{I}^{\{1,3\}}-r_{I}^{\{2,3\}}-r_{I}^{\{1,2,3\}} \equiv 0(\bmod 4), \\
r_{I}^{\{3\}}-r_{I}^{\{1,3\}}-r_{I}^{\{2,3\}}+r_{I}^{\{1,2,3\}} \equiv 0(\bmod 4) .
\end{array}\right.
\end{gathered}
$$

Определим множество векторов

$$
\begin{array}{r}
\mathfrak{R}^{\prime}=\{(0,0,0,0,0,0),(2,2,0,2,0,0),(2,0,2,0,2,0),(0,2,2,0,0,2), \\
(0,0,0,2,2,2),(0,2,2,2,2,0),(2,0,2,2,0,2),(2,2,0,0,2,2)\} .
\end{array}
$$

Путем несложных рассуждений и перебора возможных вариантов можно доказать, что решениями систем будут векторы трех видов:

1) $\overrightarrow{r_{I}}=\left(r_{I}^{\{1\}} ; r_{I}^{\{2\}} ; r_{I}^{\{3\}} ; r_{I}^{\{1,2\}} ; r_{I}^{\{1,3\}} ; r_{I}^{\{2,3\}} ; r_{I}^{\{1,2,3\}}\right)=\left(\vec{r}^{\prime} ; 0\right)$, где $\vec{r}^{\prime} \in$ $\mathfrak{R}^{\prime}$; обозначим множество таких векторов $\mathfrak{R}_{\text {I,even }, 0}$; 
2) $\vec{r}_{I}=(2 ; 2 ; 2 ; 2 ; 2 ; 2 ; 2)-\vec{r}^{\prime \prime}$, где $\vec{r}^{\prime \prime} \in \mathfrak{R}_{I, \text { even }, 0} ;$ обозначим множество таких векторов $\mathfrak{R}_{I, \text { even }, 2}$;

3) $\vec{r}_{I}=(1,1,1,1,1,1,1)+\vec{r}^{\prime \prime \prime}$, где $\vec{r}^{\prime \prime \prime} \in \mathfrak{R}_{I, \text { even }, 0} \cup \mathfrak{R}_{I, \text { even }, 2}=\mathfrak{R}_{I, \text { even }}$; обозначим множество таких векторов $\mathfrak{R}_{I, \text { odd }}$.

Пусть $\mathfrak{R}_{I}=\mathfrak{R}_{I, \text { even }} \cup \mathfrak{R}_{I, \text { odd }}$. Очевидно, $\left|\mathfrak{R}_{I}\right|=32$.

Тогда $\left|\mathfrak{R}^{* *}\right|=32^{L(n, k)}=2^{5 L(n, k)}$. Теперь рассмотрим, используя уже введенные обозначения, сумму

$$
\begin{aligned}
\sum_{\vec{r} \in \mathfrak{R}^{* *}} \exp \left[-i\left(\pi 2^{\frac{n}{2}} \vec{r}, \bar{z}\right)\right]= \\
=\sum_{\vec{r} \in \mathfrak{R}^{* *}} \exp \left[-i \frac{\pi}{2}\left(\sum_{\substack{I \subset \overline{1, n},|I| \leq k-1}} \sum_{\substack{\varnothing \neq J \subset \overline{\mid}|\leq k-| I|| I \mid}} r_{I}^{J} \cdot\left(\mathrm{E} w_{I}^{J}+z_{I}^{J} 2^{\frac{n}{2}+1}\right)\right]=\right. \\
=\sum_{\vec{r} \in \mathfrak{R}^{* *}} \prod_{I \subset \overline{1, n},|I| \leq k} \exp \left[-i \frac{\pi}{2}\left(\sum_{\varnothing \neq J \subset \overline{1,3}} r_{I}^{J} \cdot\left(\mathrm{E} w_{I}^{J}+z_{I}^{J} 2^{\frac{n}{2}+1}\right)\right)\right]= \\
=\left(\prod_{I \subset \overline{1, n},|I| \leq k} \sum_{\sum_{r_{I} \in \mathfrak{R}_{I}}} \exp \left[-i \pi\left(\sum_{\varnothing \neq J \subset \overline{1,2}} r_{I}^{J} \cdot v_{I}^{J}\right)\right]\right)
\end{aligned}
$$

Используя сравнения (1) из следствия 1 [8], несложно показать, что $\sum_{\vec{r} \in \mathfrak{R}^{* *}} \exp \left[-i \pi\left(2^{n / 2} \vec{r}, \bar{z}\right)\right]=2^{5 L(n, k)}=\left|\mathfrak{R}^{* *}\right|$.

Утверждение 3. Для мощности множества $\mathfrak{R}^{* *}(m, N)$, определенного в теореме 1 [7], справедливы двусторонние оценки

$$
\begin{aligned}
\exp _{2}((m-1) N(n, 1, k)) \leq & \left|\Re^{* *}(m, N)\right| \leq \\
& \leq \exp _{2}((m-2) N(n, m, k)+N(n, 1, k)) .
\end{aligned}
$$

Доказательство. Верхняя оценка мощности $\mathfrak{R}^{* *}$ следует из того, что при фиксированном $I$ величины $r_{I}^{J}$ имеют одинаковую четность согласно утверждению 1.

Чтобы установить нижнюю границу, рассмотрим такие векторы $\vec{r}$ из множества $\left\{0, \ldots, 2^{m-1}-1\right\}^{N}$, что $r_{I}^{J}=t_{I}$, где $t_{I} \in\left\{0, \ldots, 2^{m-1}-1\right\}$, для всех $|I| \leq k$ и для любых $\varnothing \neq J \subset \overline{1, m}$. 
Так как $\sum_{J \subset \overline{1, m}, s \in J}(-1)^{\left(\delta, \psi_{m}(J)\right) \oplus 1} r_{I}^{J} \in\left\{ \pm 2^{m-1} t_{I}, 0\right\} \in 2^{m-1} \mathbb{Z}$ для всех $s \in \overline{1, m}$ и для всех $\delta \in V_{m}$, то $\vec{r} \in \mathfrak{R}^{* *}$.

Следовательно, мощность $\left|\mathfrak{R}^{* *}\right|$ не меньше, чем число таких векторов. Значит, $\left|\mathfrak{R}^{* *}\right| \geq \exp _{2}((m-1) N(n, 1, k))$.

Верхняя и нижняя оценки из утверждения 3 при $m=2$ совпадают и, следовательно, являются достижимыми .

Следующий результат получается с помощью утверждения 10 [7] и оценок из утверждения 3.

Утверждение 4. При $k(n)=o(\sqrt{n}), m(n)=o(n)$ и любых $\varepsilon_{1}, \varepsilon_{2}>0$ существует такое $n_{0}<\infty$, что для $n>n_{0}$ верны неравенства

$$
\begin{aligned}
& \left(1-\varepsilon_{1}\right) \exp _{2}\left(m 2^{n}-T(n, m, k)+(m-1) N(n, 1, k)\right)<|R[n, m, k]|< \\
& <\left(1+\varepsilon_{2}\right) \exp _{2}\left(m 2^{n}-T(n, m, k)+(m-2) N(n, m, k)+N(n, 1, k)\right) .
\end{aligned}
$$

\section{2. Оценки для числа корреляционно-иммунных двоичных отображений}

Согласно формуле (1) из [1] компонента $\left(\psi_{m}(J), f\right)$ функции $f$ является корреляционно-иммунной порядка $k$, если существует такая величина $r_{J} \in\left\{-2^{n-k-1}, \ldots, 2^{n-k-1}\right\}$, что для любого $I$, мощность которого ограничена неравенством $0 \leq|I| \leq k$, верно равенство $w_{I}^{J}=$ $\mathrm{E} w_{I}^{J}+r_{J} 2^{k-|I|}$.

Из следствия $1[8]$ получаем, что $2^{1-|J|} \sum_{\varnothing \neq S \subset J}(-1)^{|S|} w_{I}^{S} \in \mathbb{Z}$.

Следовательно,

$$
\begin{aligned}
& 2^{1-|J|} \sum_{\varnothing \neq S \subset J}(-1)^{|S|}\left(\mathrm{E} w_{I}^{S}+r_{S} 2^{k-|I|}\right)= \\
& =2^{n-|J|-|I|} \sum_{\varnothing \neq S \subset J}(-1)^{|S|}+2^{k+1-|J|-|I|} \sum_{\varnothing \neq S \subset J}(-1)^{|S|} r_{S}= \\
& \quad=-2^{n-|I|-|J|}+2^{k-|I|-|J|+1} \sum_{\varnothing \neq S \subset J}(-1)^{|S|} r_{S} \in \mathbb{Z} .
\end{aligned}
$$

Включение $2^{k-|I|-|J|+1} \sum_{\varnothing \neq S \subset J}(-1)^{|S|} r_{S} \in \mathbb{Z}$ должно выполняться для всех таких $I$, что $|I| \leq k$. Достаточно, чтобы оно выполнялось при $|I|=k$. 
Отсюда вытекает следующее отношение сравнимости, которому должны удовлетворять все допустимые значения $r_{J} \in\left\{-2^{n-k-1}, \ldots, 2^{n-k-1}\right\}$ при достаточно больших $n$ :

$$
r_{J} \equiv \sum_{\varnothing \neq S \subset J, S \neq J}(-1)^{|J \backslash S|+1} r_{S}\left(\bmod 2^{|J|-1}\right) .
$$

Из этого сравнения следует, что вычет $r_{J}$ по модулю $2^{|J|-1}$ при фиксированном наборе значений $r_{S}$ для всех $\varnothing \neq S \subset J, S \neq J$, определяется однозначно.

Вероятность того, что любая ненулевая комбинация $f^{J}$ координатных функций функции $f$ является корреляционно-иммунной порядка $k$, равна

$$
\mathrm{P}\{f \in K[n, m, k]\}=\sum_{\substack{r_{J} \in\left\{-2^{n-k-1}, \ldots, 2^{n-k-1}\right\}, \varnothing \neq J \subset \overline{1, m} \\ r_{J} \equiv \sum_{\varnothing \neq S \subset J, S \neq J}(-1)^{|J \backslash S|+1} r_{S}\left(\bmod 2^{|J|-1}\right)}} \mathrm{P}\left\{\bar{w}-\mathrm{E} \bar{w}=\bar{r}_{\bar{z}} 2^{\frac{n}{2}+m-2}\right\},
$$

где $K[n, m, k]$ - множество функций, любая ненулевая комбинация координатных функций которых является корреляционно-иммунной порядка $k, \bar{r} \bar{z}=\left(r_{J} \cdot z^{J}, \varnothing \neq J \subset \overline{1, m}\right), z_{I}^{J}=2^{k-m-|I|-n / 2+2}$ для всех таких $I$, что $0 \leq|I| \leq k$.

Теорема 5. Пусть $n \rightarrow \infty, k(n)=o(\sqrt{n}), m(n)=o(n)$. Тогда для любого $\varepsilon>0$ верно неравенство

$$
\begin{aligned}
|K[n, m, k]|<\exp _{2}\left(m\left(2^{n}-2^{m-1}\right)+\left(2^{m}-1\right)(n-k+1)-\frac{n}{2}+\right. \\
\left.-T(n, m, k)+3 m+(3 k+3+\varepsilon) \log _{2} n\right)\left|\mathfrak{R}^{* *}(m, N)\right| .
\end{aligned}
$$

Доказательство. Из теоремы 1 [7] следует, что верно неравенство

$$
\begin{aligned}
\mathrm{P}\left\{\bar{w}-\mathrm{E} \bar{w}={ }_{\bar{r}} \bar{z} 2^{n / 2+m-2}\right\} \leq & \\
& \leq\left|\mathfrak{R}^{* *}(m, N)\right| \frac{\exp \left(-\frac{1}{2} \bar{r}^{T} \bar{z}^{T} Q^{-1} \bar{r} \bar{z}\right)+O\left(n^{3 k+3} 2^{3 m-n / 2}\right)}{2^{\frac{n+2 m-3}{2} N} \sqrt{\pi^{N} \operatorname{det} Q}} .
\end{aligned}
$$

Также из теоремы 1 [7], проводя доказательство аналогично лемме 6 [7], 
и из утверждения 10 [7] можно вывести, что

$$
\begin{aligned}
& \frac{|K[n, m, k]|}{\left|B_{n}^{m}\right|}=\mathrm{P}\{f \in K[n, m, k]\}=\sum \mathrm{P}\left\{\bar{w}-\mathrm{E} \bar{w}=\bar{r} \bar{z} 2^{\frac{n}{2}+m-2}\right\} \leq \\
& \leq \sum \frac{|R[n, m, k]|}{\left|B_{n}^{m}\right|}\left(\exp \left(-\frac{1}{2}(\bar{r} \bar{z})^{T} Q(\bar{r} \bar{z})\right)+O\left(n^{3 k+3} 2^{-\frac{n}{2}+3 m}\right)\right)= \\
& \quad \sum \frac{|R[n, m, k]|}{\left|B_{n}^{m}\right|}\left(\exp \left(-\frac{1}{2} \sum_{\varnothing \neq J \subset \overline{1, m}}\left(r_{J}\right)^{2}\left(z^{J}\right)^{T} Q_{J}\left(z^{J}\right)\right)+\right. \\
& \left.+O\left(n^{3 k+3} 2^{-\frac{n}{2}+3 m}\right)\right)=\sum \exp \left(-\frac{1}{2} \sum_{\varnothing \neq J \subset \overline{1, m}}\left(r_{J}\right)^{2}\left(z^{J}\right)^{T} Q_{J}\left(z^{J}\right)\right) \times \\
& \times \frac{|R[n, m, k]|}{\left|B_{n}^{m}\right|}+O\left(n^{3 k+3} 2^{-\frac{n}{2}+3 m}\right) \frac{|R[n, m, k]|}{\left|B_{n}^{m}\right|} \sum 1 .
\end{aligned}
$$

Суммирования в данной цепочке неравенств проводятся по всем непустым $J \subset \overline{1, m}$, по всем $r_{J}$ из множества $\left\{-2^{n-k-1}, \ldots, 2^{n-k-1}\right\}$, для которых выполняется условие $r_{J} \equiv \sum_{\varnothing \neq S \subset J, S \neq J}(-1)^{|J \backslash S|+1} r_{S}\left(\bmod 2^{|J|-1}\right)$.

Проводя доказательство аналогично лемме 7 [7] для любых $\varnothing \neq J \subset \overline{1, m}$, получаем

$$
\begin{gathered}
\left(z^{J}\right)^{T} Q_{J}^{-1}\left(z^{J}\right)=2^{2 m-2} \sum_{I \subset \overline{1, n}|I| \leq k}\left(\sum_{S \subset I}(-1)^{|S|} 2^{|S|} z_{S}^{J}\right)^{2}= \\
=2^{2 m-2} \sum_{I \subset \overline{1, n},|I| \leq k}\left(\sum_{S \subset I}(-1)^{|S|} 2^{|S|} 2^{k-m-|S|-\frac{n}{2}+2}\right)^{2}= \\
=2^{2 k+2-n} \sum_{I \subset \overline{1, n},|I| \leq k}\left(\sum_{S \subset I}(-1)^{|S|}\right)^{2}= \\
2^{2 k+2-n} \times \sum_{i=0}^{k}\left(\begin{array}{c}
n \\
i
\end{array}\right)\left(\begin{array}{c}
i \\
\left.(-1)^{i} \sum_{s=0}\left(\begin{array}{c}
i \\
s
\end{array}\right)(-1)^{s}\right)^{2}=2^{2 k-n+2} .
\end{array}\right.
\end{gathered}
$$

Данное равенство позволяет преобразовать одно из получившихся выше 
выражений следующим образом:

$$
\begin{aligned}
& \sum_{\varnothing \neq J \subset \overline{1, m},} \exp \left(-\frac{1}{2} \sum_{\varnothing \neq J \subset \overline{1, m}}\left(r_{J}\right)^{2}\left(z^{J}\right)^{T} Q_{J}\left(z^{J}\right)\right)= \\
& r_{J} \in\left\{-2^{n-k-1}, \ldots, 2^{n-k-1}\right\}, \\
& r_{J} \equiv \sum_{\substack{\varnothing \neq S \subset J, S \neq J}}(-1)^{|J \backslash S|+1} r_{S}\left(\bmod 2^{|J|-1}\right) \\
& =\sum_{\substack{\varnothing \neq J \subset \overline{1, m}, J \neq \overline{1, m}, r_{J} \in\left\{-2^{n-k-1}, \ldots, 2^{n-k-1}\right\},}} \exp \left(-\frac{1}{2} \sum_{\substack{\varnothing \neq J \subset \overline{1, m}, J \neq \overline{1, m}}}\left(r_{J}\right)^{2} 2^{2 k-n+2}\right) \times \\
& r_{J} \equiv \sum_{\substack{\varnothing \neq S \subset J, S \neq J}}(-1)^{|J \backslash S|+1} r_{S}\left(\bmod 2^{|J|-1}\right) \\
& \times \quad \sum_{n=\{} \quad \exp \left(-\left(r_{\overline{1, m}}\right)^{2} 2^{2 k-n+1}\right) .
\end{aligned}
$$

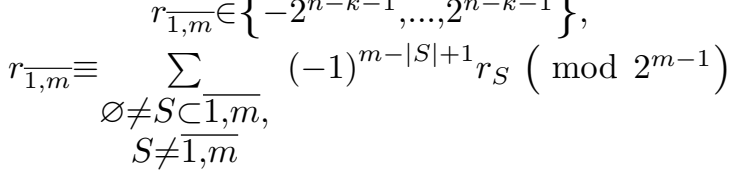

Зафиксируем $r_{S}$ по всем $\varnothing \neq S \subset \overline{1, m}, S \neq \overline{1, m}$, и рассмотрим сумму

$$
\begin{aligned}
& \sum_{n} \exp \left(-\left(r_{1, m}\right)^{2} 2^{2 k-n+1}\right) \\
& r_{\overline{1, m}} \in\left\{-2^{n-k-1}, \ldots, 2^{n-k-1}\right\} \\
& r_{\overline{1, m}} \equiv \sum_{\substack{\varnothing \neq S \subset \overline{1, m} \\
S \neq \overline{1, m}}}(-1)^{m-|S|+1} r_{S}\left(\bmod 2^{m-1}\right)
\end{aligned}
$$

Из того, что $r_{\overline{1, m}} \equiv \sum_{\varnothing \neq S \subset \overline{1, m}}(-1)^{m-|S|+1} r_{S}\left(\bmod 2^{m-1}\right)$, следует, что существует такое $k \in \frac{S \neq \overline{1, m}}{0,2^{m-1}-1}$, что $r_{\overline{1, m}} \equiv k\left(\bmod 2^{m-1}\right)$.

Пусть $k=0$. Тогда $r_{\overline{1, m}}=2^{m-1} t$, и суммирование проводится по всем 
возможным целочисленным Значениям $t$ от $-2^{n-k-m}$ до $2^{n-k-m}$ :

$$
\begin{aligned}
& \sum_{\substack{r_{\overline{1, m}} \in\left\{-2^{n-k-1}, \ldots, 2^{n-k-1}\right\}, r_{1, m}=0\left(\bmod 2^{m-1}\right)}} \exp \left(-\left(r_{\overline{1, m}}\right)^{2} 2^{2 k-n+1}\right)= \\
& =\sum_{t=-2^{n-k-m}}^{2^{n-k-m}} \exp \left(-\frac{\left(2^{m-1} t\right)^{2}}{2} 2^{2 k-n+2}\right) .
\end{aligned}
$$

Если сделать замену $x_{t}=2^{m+k-\frac{n}{2}} t$, то получаем равенство

$$
\begin{aligned}
& \sum_{t=-2^{n-k-m}}^{2^{n-k-m}} \exp \left(-\frac{\left(2^{m-1} t\right)^{2}}{2} 2^{2 k-n+2}\right)= \\
& =2^{-m-k+\frac{n}{2}} \sum_{x_{t}} \exp \left(-\frac{\left(x_{t}\right)^{2}}{2}\right)\left(x_{t+1}-x_{t}\right) .
\end{aligned}
$$

Сумма $\sum_{x_{t}} \exp \left(-\frac{\left(x_{t}\right)^{2}}{2}\right)\left(x_{t+1}-x_{t}\right)$ есть интеграл от ступенчатой функции в пределах от $-2^{\frac{n}{2}}$ до $2^{\frac{n}{2}}$, который при $n \rightarrow \infty$ сходится к интегралу $\int_{-\infty}^{\infty} \exp \left(-\frac{t^{2}}{2}\right) d t=\sqrt{2 \pi}$. Следовательно, при $n \rightarrow \infty$

$$
\sum_{t_{S}=-2^{n-k-m}}^{2^{n-k-m}} \exp \left(-\frac{\left(2^{m-1} t_{\overline{1, m}}\right)^{2}}{2} 2^{2 k-n+2}\right) \sim 2^{n / 2-k-m} \sqrt{2 \pi}
$$

Такая же асимптотика получается для всех возможных значений $k \in$ $\overline{1,2^{m-1}-1}$. Следовательно,

$$
\sum_{\substack{\varnothing \neq J \subset \overline{1, m}, r_{J} \in\left\{2^{n-k-1}, \ldots, 2^{n-k-1}\right\},}} \exp \left(\sum_{\substack{\varnothing \neq S \subset J,-S \neq J}}-\frac{\left(r_{J}\right)^{2}}{2}\left(z^{J}\right)^{T} Q_{J}\left(z^{J}\right)\right) \sim
$$




$$
\sim 2^{\frac{n}{2}-k-m \sqrt{2 \pi}} \sum_{\substack{\varnothing \neq J \subset \overline{1, m}, J \neq \overline{1, m}, r_{J} \in\left\{-2^{n-k-1}, \ldots, 2^{n-k-1}\right\} \\
r_{J} \equiv \sum_{\begin{subarray}{c}{\varnothing \neq S \subset J, S \neq J} }}(-1)^{|J \backslash S|+1} r_{S}}\end{subarray}} \exp \left(\sum_{\substack{\varnothing \neq J \subset \overline{1, m}, J \neq \overline{1, m}}}-\frac{\left(r_{J}\right)^{2}}{2} 2^{2 k-n+2}\right) .
$$

Теперь последовательно повторяем рассуждения для всех $J \subset \overline{1, m}, 1 \leq$ $|J| \leq m-1$, начиная с множеств большей мощности. В итоге получаем

$$
\begin{aligned}
& \sum_{\varnothing \neq J \subset \overline{1, m}, r_{J} \in\left\{-2^{n-k-1}, \ldots, 2^{n-k-1}\right\},} \exp \left(\sum_{\varnothing \neq J \subset \overline{1, m}}-\frac{\left(r_{J}\right)^{2}}{2}\left(z^{J}\right)^{T} Q_{J}\left(z^{J}\right)\right) \sim \\
& r_{J} \equiv \sum_{\substack{\varnothing \neq S \subset J, S \neq J}}(-1)^{|J \backslash S|+1} r_{S}\left(\bmod 2^{|J|-1}\right) \\
& \sim \prod_{\varnothing \neq J \subset \overline{1, m}}\left(2^{n / 2-k-|J|} \sqrt{2 \pi}\right)=\left(2^{n / 2-k} \sqrt{2 \pi}\right)^{2^{m}-1} \prod_{\varnothing \neq J \subset \overline{1, m}} 2^{-|J|}= \\
& =\exp _{2}\left(\left(\frac{n+1+\log _{2} \pi}{2}-k\right)\left(2^{m}-1\right)-m 2^{m-1}\right) .
\end{aligned}
$$

Дословно повторяя уже проведенные этапы рассуждений, можно показать, что

$$
\begin{gathered}
\sum_{\varnothing \neq J \subset \overline{1, m}, r_{J} \in\left\{-2^{n-k-1}, \ldots, 2^{n-k-1}\right\},} 1 \sim \prod_{\varnothing \neq J \subset \overline{1, m}} 2^{n-k-|J|+1}= \\
r_{J} \equiv \sum_{\varnothing \neq S \subset J, S \neq J}(-1)^{|J \backslash S|+1} r_{S}\left(\bmod 2^{|J|-1}\right) \\
=2^{(n-k+1)\left(2^{m}-1\right)-m 2^{m-1}} .
\end{gathered}
$$

Следовательно,

$$
\begin{aligned}
& \frac{|R[n, m, k]|}{\left|B_{n}^{m}\right|}\left(2^{\left(\frac{n+1+\log _{2} \pi}{2}-k\right)\left(2^{m}-1\right)-m 2^{m-1}}+O\left(n^{3 k+3} 2^{-n / 2+3 m}\right) 2^{-m 2^{m-1}} \times\right. \\
&\left.\times 2^{(n-k+1)\left(2^{m}-1\right)}\right)=O\left(\frac{|R[n, m, k]|}{\left|B_{n}^{m}\right|} n^{3 k+3} 2^{(n-k+1)\left(2^{m}-1\right)-m 2^{m-1}-n / 2+3 m}\right) .
\end{aligned}
$$

Из этого равенства следует формулировка теоремы.

Согласно доказательству утверждения 2 при $m \in\{2,3\}$ выполняется равенство $\left|\mathfrak{R}^{* *}(m, N)\right|=2^{N(n, 1, k)(4 m-7)}$. Это обосновывает следующее утверждение 
Следствие 6. Пусть $n \rightarrow \infty, k(n)=o(\sqrt{n}), m \in\{2,3\}$. Тогда для любого $\varepsilon>0$ верно неравенство

$$
\begin{aligned}
& |K[n, m, k]|<\exp _{2}\left(m\left(2^{n}-2^{m-1}\right)+\left(2^{m}-1\right)(n-k+1)-\frac{n}{2}+\right. \\
& \left.\quad-T(n, m, k)+3 m+(3 k+3+\varepsilon) \log _{2} n+N(n, 1, k)(4 m-7)\right) .
\end{aligned}
$$

Следствие 7. Пусть $n \rightarrow \infty, k(n)=o(\sqrt{n}), m(n)=o(n)$. Тогда для любого $\varepsilon>0$ верно неравенство

$$
\begin{aligned}
& |K[n, m, k]|<\exp _{2}\left(m\left(2^{n}-2^{m-1}\right)+\left(2^{m}-1\right)(n-k+1)-\frac{n}{2}+\right. \\
& \left.-T(n, m, k)+3 m+(3 k+3+\varepsilon) \log _{2} n+(m-2) N+N(n, 1, k)\right) .
\end{aligned}
$$

Доказательство следствия 7 следует из утверждения 3.

Нижней оценкой для числа корреляционно-иммунных порядка $k$ отображений может служить нижняя оценка числа $k$-эластичных отображений, полученная в утверждении 4.

Случай $m=1$ рассматривается аналогично следствию 2 [2].

Утверждение 8. Пусть $n \rightarrow \infty, k(n)=o(\sqrt{n})$. Тогда

$$
\begin{aligned}
&|K[n, 1, k]| \sim|R[n, 1, k]|\left(\pi 2^{n-2 k-1}\right)^{\frac{1}{2}} \sim \\
& \sim \exp _{2}\left(2^{n}-T(n, 1, k)+\frac{n}{2}-k+\log _{2} \sqrt{\frac{\pi}{2}}\right) .
\end{aligned}
$$

Утверждение 8 соответствует результату, доказанному в [1] для любого фиксированного $k$, исправляет неточность, допущенную в [2] для $k(n)=o(\sqrt{n})$, и является частным случаем результата, полученного в [18] для $k \leq\left(\frac{\ln 2}{6}-\varepsilon\right) \frac{n}{\ln n}$ при любом фиксированном $\varepsilon>0$.

Полученные в этом параграфе результаты согласуются с точными верхней и нижней оценками числа корреляционно-иммунных булевых функций 1-го порядка, полученными в [14].

\section{3. Оценки для числа платовидных двоичных отображений фиксированного порядка}

Рассмотрим вектор

$$
\bar{F}=\bar{F}(f, n, m, k)=\left(\Delta_{I}^{J}(f), 0 \leq|I| \leq k, I \subset \overline{1, n}, \varnothing \neq J \subset \overline{1, m}\right)
$$


размерности $N(n, m, k)$, состоящий из части коэффициентов статистической структуры всех компонент отображения $f$ и имеющий такую же структуру, как $\bar{w}$.

Согласно следствию 2 [8] коэффициенты статистической структуры компонент двоичного отображения удовлетворяют следующим сравнениям:

$$
\begin{gathered}
\sum_{L \subset I}(-1)^{|L|} \Delta_{L}^{J} \equiv 0\left(\bmod 2^{|I|}\right), \\
\sum_{\varnothing \neq S \subset J, L \subset I}(-1)^{|L|+|S|} \Delta_{L}^{S} \equiv 0\left(\bmod 2^{|I|+|J|-1}\right) .
\end{gathered}
$$

Докажем для вектора $\bar{F}(f, n, m, k)$ теорему, аналогичную теореме 1 [7] для вектора $\bar{w}(n, m, k)$.

Везде далее $[[\bar{a}]]$ - евклидова норма вектора $\bar{a}$.

Теорема 9. Пусть $n \rightarrow \infty, k(n)=o(\sqrt{n}), m(n)=o(n)$. Тогда равномерно относительно векторов $\bar{a}$ размерности $N(n, m, k)$, координаты которых удовлетворяют сравнениям (4) и (5), справедливо представление

$$
\begin{aligned}
& \mathrm{P}(\bar{F}=\bar{a})=\frac{\exp \left(-2^{-n+1}[[\bar{a}]]^{2}\right)+O\left(n^{3 k+3} 2^{-\frac{n}{2}+3 m}\right)}{\exp _{2}(T(n, m, k))} \times \\
& \quad \times \sum_{\vec{r} \in \mathfrak{R}^{* *}(m, N)} \exp \left[-i \pi \sum_{\varnothing \neq J \subset \overline{1, m}} \sum_{I \subset 1, n,|I| \leq k} 2^{\frac{n}{2}-|I|} r_{I}^{J} \sum_{L \subset I}(-1)^{|L|+1} a_{L}^{J}\right],
\end{aligned}
$$

где $\bar{a}$ имеет такую же структуру координат как $\bar{F}$, а множество $\mathfrak{R}^{* *}(m, N)$ определено выше (см. (2)).

Доказательство. Из теоремы 1 [7] и формул связи между весами подфункций компонент функции и их коэффициентами статструктуры

$$
\begin{gathered}
\Delta_{I}^{J}=\sum_{L \subset I}(-1)^{|L|}\left(2^{n-1}-2^{|L|} w_{L}^{J}\right), \\
w_{I}^{J}-2^{n-|I|-1}=2^{-|I|} \cdot \sum_{L \subset I}(-1)^{|L|+1} \Delta_{L}^{J},
\end{gathered}
$$

доказанных в [2], получаем, что при $n \rightarrow \infty$

$$
\mathrm{P}(\bar{F}=\bar{a})=\mathrm{P}(\bar{w}-\mathrm{E} \bar{w}=\bar{z}(\bar{a}))=
$$




$$
\begin{array}{r}
=\frac{\exp \left(-\left(\bar{z}(\bar{a})^{T}(Q(n, m, k))^{-1} \bar{z}(\bar{a})\right) 2^{-n-2 m+3}\right)+O\left(n^{3 k+3} 2^{-\frac{n}{2}+3 m}\right)}{\exp _{2}(}(T(n, m, k)) \\
\times\left(\sum_{\vec{r} \in \mathfrak{R}^{* *}} \exp \left[-i \pi\left(2^{\frac{n}{2}} \vec{r}, \bar{z}(\bar{a})\right)\right]\right)
\end{array}
$$

равномерно относительно таких $\bar{a}$, что вектор $\bar{z}(\bar{a})$ с компонентами

$$
z_{I}^{J}(\bar{a})=2^{-|I|} \sum_{L \subset I}(-1)^{|L|+1} a_{L}^{J}
$$

целочисленный, что вытекает из сравнения (4).

Согласно сравнению (5) для любых $I, J, \varnothing \neq J \subset \overline{1, m}, I \subset$ $\overline{1, n}, 1 \leq|I|+|J| \leq k$, целыми числами являются выражения $2^{-|I|-|J|+1} \sum_{\varnothing \neq S \subset J} \sum_{L \subset I}(-1)^{|L|+|S|} a_{L}^{S}$, что равносильно сравнениям (1) для весов подфункций, так как

$$
\begin{aligned}
& 2^{-|I|-|J|+1} \sum_{\varnothing \neq S \subset J} \sum_{L \subset I}(-1)^{|L|+|S|} a_{L}^{S}= \\
= & 2^{1-|J|} \sum_{\varnothing \neq S \subset J}(-1)^{|S|} 2^{-|I|} \sum_{L \subset I}(-1)^{|L| s} a_{L}^{S}=2^{1-|J|} \sum_{\varnothing \neq S \subset J}(-1)^{|S|} z_{I}^{S}(\bar{a}) .
\end{aligned}
$$

Рассмотрим теперь

$$
\begin{gathered}
\bar{z}(\bar{a})^{T}(Q(n, m, k))^{-1} \bar{z}(\bar{a})= \\
=2^{2 m-2} \sum_{\substack{J \subset \overline{1, m}, J \neq \varnothing}} \sum_{\substack{I \subset \overline{1}, n \\
|I| \leq k}}\left(\sum_{K \subset I}(-1)^{|K|} 2^{|K|} z_{K}^{J}\right)^{2}= \\
=2^{2 m-2} \sum_{\substack{J \subset \overline{1, m}, I \subset \overline{1, n}, J \neq \varnothing}} \sum_{\substack{|I| \leq k \\
K}}\left(\sum_{L \subset I}(-1)^{|L|+1} a_{L}^{J} \sum_{L \subset K \subset I}(-1)^{|K|}\right)^{2},
\end{gathered}
$$

где сумма $\sum_{L \subset K \subset I}(-1)^{|K|}$ равна $(-1)^{|L|}$, если $I=L$, и равна нулю в противном случае.

Следовательно,

$$
\bar{z}(\bar{a})^{T}(Q(n, m, k))^{-1} \bar{z}(\bar{a})=2^{2 m-2} \sum_{\substack{J \subset \overline{1, m}, I \subset \overline{1, n}, J \neq \varnothing}} \sum_{\substack{|I| \leq k \\ \text { a }}}\left(a_{I}^{J}\right)^{2} .
$$


Далее,

$$
\begin{gathered}
\sum_{\vec{r} \in \mathfrak{R}^{* *}} \exp \left[-i \pi \quad\left(2^{\frac{n}{2}} \vec{r}, \bar{z}(\bar{a})\right)\right]= \\
=\sum_{\vec{r} \in \mathfrak{R}^{* *}} \exp \left[-i \pi 2^{\frac{n}{2}} \sum_{\substack{J \subset \bar{c}, m, J \neq \varnothing}} \sum_{\substack{|\subset \overline{1, n},\\
| I \mid \leq k}} r_{I}^{J} \cdot z_{I}^{J}(\bar{a})\right]= \\
=\sum_{\vec{r} \in \mathfrak{R}^{* *}} \exp \left[-i \pi \sum_{\substack{J \subset \overline{1, m}, I \subset \overline{1, n}, J \neq \varnothing}} \sum_{\substack{|I| \leq k \\
n^{2}}} 2^{\frac{n}{2}-|I|} r_{I}^{J} \sum_{L \subset I}(-1)^{|L|+1} a_{L}^{J}\right] .
\end{gathered}
$$

Теорема доказана.

Следствие 10. В условиях теоремы 9 равномерно относительно векторов а размерности $N(n, m, k)$, удовлетворяющих сравнениям (4) $u$ (5) и соотношению

$$
[[\bar{a}]]^{2}=o\left(2^{n-2}((n-6 m) \ln 2+(6-6 k) \ln n)\right),
$$

справедливо неравенство

$$
\mathrm{P}(\bar{F}=\bar{a}) \leq \frac{\left(1+\varepsilon_{n}\right) \exp \left(-2^{-n+1}[[\bar{a}]]^{2}\right)}{\exp _{2}(T(n, m, k))}\left|\mathfrak{R}^{* *}\right|, \varepsilon_{n} \rightarrow 0, \text { nрu } n \rightarrow \infty,
$$

а если $[[\bar{a}]]^{2} \neq o\left(2^{n-2}((n-6 m) \ln 2+(6-6 k) \ln n)\right)$, mo

$$
\mathrm{P}(\bar{F}=\bar{a})=O\left(n^{3 k+3} 2^{-\frac{n}{2}-T}(n, m, k)+3 m\right)\left|\mathfrak{R}^{* *}\right| .
$$

Обозначим через $\Pi\left(r_{J}, \varnothing \neq J \subset \overline{1, m}\right)$ множество всех платовидных двоичных отображений порядка $\left(r_{J}, \varnothing \neq J \subset \overline{1, m}\right)$.

Теорема 11. Пусть $n \rightarrow \infty, m(n)=o(n)$. Тогда для любого $k(n)=$ o $(\sqrt{n})$ для любого $\varepsilon>0$ мощность множества $\Pi\left(r_{J}, \varnothing \neq J \subset \overline{1, m}\right)$, где $\max _{J}\left(\frac{n}{2}-r_{J}\right)=o(\ln n)$, ограничена сверху выражением

$$
\exp _{2}\left(m 2^{n}-T(n, m, k)+N(n, m, k) \log _{2} 3+\varepsilon \log _{2} n\right)\left|\Re^{* *}(m, N)\right| .
$$


Доказательство. Оценим вероятность того, что случайное двоичное отображение является платовидным порядка $\left(r_{J}, \varnothing \neq J \subset \overline{1, m}\right)$. Обозначим $t_{J}=\frac{n}{2}-r_{J}$. При этом $\max _{J}\left(t_{J}\right)=o(\ln n)$.

Очевидно, что вероятность этого события не превышает вероятности того, что в неполном векторе коэффициентов статструктуры размерности $N(n, m, k)$ все соответствующие координаты равны либо $\pm 2^{n / 2+t_{J}-1}$, либо 0. Обозначим последнее событие через П $(\Delta)$ и используем теоремy 9 :

$$
\mathrm{P}\left(\Pi\left(r_{J}, \varnothing \neq J \subset \overline{1, m}\right)\right) \leq \mathrm{P}(\Pi(\Delta)) .
$$

При $t_{J}=o(\ln n)$ величина $L=L(n, k)=N(n, 1, k)$ из определения $N(n, m, k)$ меньше, чем $2^{r_{J}}$, и данные неполные векторы удовлетворяют отношениям сравнимости для коэффициентов статистической структуры.

Пусть в соответствующем неполном наборе коэффициентов статистической структуры в подвекторах, соответствующих линейным комбинациям, сопоставленных множествам $J$, будет ровно $s_{J}$ ненулевых координат. Тогда $[[\bar{a}]]^{2}=2^{n-2} \sum_{\varnothing \neq J \subset \overline{1, m}} s_{J} 2^{2 t_{J}}$. Каждая из этих координат может быть как положительной, так и отрицательной. Количество же мест, на которых они могут располагаться, равно $\left(\begin{array}{l}L \\ s_{J}\end{array}\right)$. Поэтому из теоремы 9 следует, что верно неравенство

$$
\begin{aligned}
\mathrm{P}(\Pi(\Delta)) \leq \sum_{\left(s_{J}, \varnothing \neq J \subset \overline{1, m}\right) \in(\overline{0, L})^{2^{m_{-}}}}\left(\prod_{\varnothing \neq J \subset \overline{1, m}} 2^{s_{J}}\left(\begin{array}{c}
L \\
s_{J}
\end{array}\right)\right) \times \\
\quad \times \frac{\exp \left(-\sum_{\varnothing \neq J \subset \overline{1, m}} s_{J} 2^{2 t_{J}-1}\right)+O\left(n^{3 k+3} 2^{-n / 2+3 m}\right)}{\exp _{2}(T(n, m, k))}\left|\mathfrak{R}^{* *}(m, N)\right| .
\end{aligned}
$$

Рассмотрим множество

$$
\begin{aligned}
S_{0}=\left\{\left(s_{J}, \varnothing \neq J \subset \overline{1, m}\right) \in(\overline{0, L})^{2^{m}-1}:\right. \\
\\
\left.\sum_{\varnothing \neq J \subset \overline{1, m}} s_{J} 2^{2 t_{J}}=o((n-6 m) \ln 2+(6-6 k) \ln n)\right\}
\end{aligned}
$$

и обозначим $S_{1}=(\overline{0, L})^{2^{m}-1} \backslash S_{0}$. Из следствия 10 получаем, что при 


$$
\begin{aligned}
& \varepsilon_{n} \rightarrow 0 \text { верно неравенство } \\
& \mathrm{P}(\Pi(\Delta)) \leq \\
& \leq\left(\sum_{\left(s_{J}, \varnothing \neq J \subset \overline{1, m}\right) \in S_{0}}\left(\prod_{\varnothing \neq J \subset \overline{1, m}} 2^{s_{J}}\left(\begin{array}{c}
L \\
s_{J}
\end{array}\right) \exp \left(-s_{J} 2^{2 t_{J}-1}\right)\right)\left(1+\varepsilon_{n}\right)+\right. \\
& \left.+\sum_{\left(s_{J}, \varnothing \neq J \subset \overline{1, m}\right) \in S_{1}}\left(\prod_{\varnothing \neq J \subset \overline{1, m}} 2^{s_{J}}\left(\begin{array}{c}
L \\
s_{J}
\end{array}\right)\right) O\left(n^{3 k+3} 2^{-\frac{n}{2}+3 m}\right)\right) \frac{\left|\mathfrak{R}^{* *}(m, N)\right|}{\exp _{2}(T(n, m, k))} \leq \\
& \leq\left(\prod_{\varnothing \neq J \subset \overline{1, m}}\left(\sum_{s_{J}=0}^{L} 2^{s_{J}}\left(\begin{array}{c}
L \\
s_{J}
\end{array}\right) \exp \left(-s_{J} 2^{2 t_{J}-1}\right)\right)\left(1+\varepsilon_{n}\right)+\right. \\
& \left.+O\left(n^{3 k+3} 2^{-\frac{n}{2}+3 m}\right) \prod_{\varnothing \neq J \subset \overline{1, m}}\left(\sum_{s_{J}=0}^{L} 2^{s_{J}}\left(\begin{array}{c}
L \\
s_{J}
\end{array}\right)\right)\right) \frac{\left|\mathfrak{R}^{* *}(m, N)\right|}{\exp _{2}(T(n, m, k))}= \\
& =\left(\prod_{\varnothing \neq J \subset \overline{1, m}}\left(\left(1+2 \exp \left(-2^{2 t_{J}-1}\right)\right)^{L}\right)\left(1+\varepsilon_{n}\right)+\right. \\
& \left.+3^{L\left(2^{m}-1\right)} O\left(n^{3 k+3} 2^{-\frac{n}{2}+3 m}\right)\right) \frac{\left|\mathfrak{R}^{* *}(m, N)\right|}{\exp _{2}(T(n, m, k))} \leq \\
& =\frac{\left|\Re^{* *}(m, N)\right| 3^{N}}{\exp _{2}(T(n, m, k))}\left(1+\varepsilon_{n}+O\left(n^{3 k+3} 2^{-\frac{n}{2}+3 m}\right)\right) \text {. }
\end{aligned}
$$

Следовательно, $\mathrm{P}(\Pi(\Delta))=O\left(\frac{3^{N(n, m, k)}\left|\mathfrak{R}^{* *}(m, N)\right|}{\exp _{2}(T(n, m, k))}\right)$.

Для ограничения сверху мощности множества $\mathfrak{R}^{* *}(m, N)$ можно воспользоваться либо утверждением 2 при $m \in \overline{2,3}$, либо утверждением 3 при больших значениях $m$.

Учитывая, что $\left|\mathfrak{R}^{* *}(m, N)\right|=1$ при $m=1$, с помощью теоремы 9 из [7] получаем следующее утверждение

Утверждение 12. Для любого $\varepsilon \in\left(0 ; \frac{1}{2}\right)$ число платовидных двоичных функииц $f \in B_{n}^{1}$ порядка $2 r$, где $\frac{n}{2}-r=o(\ln n)$, начиная с некоторого 
натурального $n$, ограничено сверху выражением

$$
\begin{aligned}
& \exp _{2}\left(2^{n}-\frac{\left(n-\left[n^{-\varepsilon+1 / 2}\right]\right)}{2}\left(\begin{array}{c}
n \\
{\left[n^{-\varepsilon+1 / 2}\right]}
\end{array}\right)-n+\right. \\
& \left.\quad+N\left(n, 1,\left[n^{-\varepsilon+1 / 2}\right]\right) \log _{2} \sqrt{\frac{18}{\pi}}+\left(5\left[n^{-\varepsilon+1 / 2}\right]+4\right) \log _{2} n\right)
\end{aligned}
$$

где $[a]-$ иелая часть а.

\section{Список литературы}

[1] Денисов О. В., “Асимптотическая формула для числа корреляционно-иммунных порядка $k$ булевых функций”, Дискретная математика, 3:2 (1991), 25-46.

[2] Денисов О. В., “Локальная предельная теорема для распределения части спектра случайной двоичной функции”, Дискретная математика, 12:1 (2000), 82-95.

[3] Зубов А.Ю., Математика кодов аутентификации, М. : Гелиос АРВ, 2007, 480 с.

[4] Иванов А.В., "Мономиальные приближения платовидных функций”, Прикл. дискр. матем., 1:1 (2008), 10-14.

[5] Кузнецов Ю.В., Шкарин С.А., “Коды Рида - Маллера", Матем. вопр. киберн., 1996, № $6,5-50$.

[6] Логачев О.А., Сальников А.А., Смышляев С.В., Ященко В.В., Булевы функции в теории кодирования и криптологии, М. : МЦНМО, 2012, 584 с.

[7] Панков К.Н., “Локальная предельная теорема для распределения части вектора весов подфункций компонент случайного двоичного отображения", Математические вопросы криптографии, 5:3 (2014), 49-80.

[8] Панков К.Н., “Оценки скорости сходимости в предельных теоремах для совместных распределений части характеристик случайных двоичных отображений", Прикл. дискр. матем., 5:4 (2012), 14-30.

[9] Сачков В.Н., Курс комбинаторного анализа, Ижевск: НИЦ “Регулярная и хаотическая динамика", 2013, 336 с.

[10] Словарь криптографических терминов, М. : МЦНМО, 2006, 94 с.

[11] Таранников Ю.В., "Комбинаторные свойства дискретных структур и приложения к криптологии", 2011, 152 с.

[12] Таранников Ю.В., "О корреляционно-иммунных и устойчивых булевых функциях", $M a-$ тем. вопр. киберн., 2002, №11, 91-148.

[13] Ященко В.В., “Свойства булевых отображений, сводимые к свойствам их координатных функций", Вестник МГУ, сер. Матем., 33:1 (1997), 11-13.

[14] Bach E., "Improved asymptotic formulas for counting correlation immune Boolean functions", SIAM J. Discrete Math., 23 (2009), 1525-1538.

[15] Bennett C., Brassard G., Robert J., "Privacy amplification by public discussion", SIAM J. Comput., 17 (1988), 210-229.

[16] Carlet C., "Vectorial Boolean functions for cryptography", Encycl. Math. and its Appl., 134 (2010), 780 pp. 
[17] Cheon J., Chee S., "Elliptic curves and resilient function", Information Security and Cryptology. - ICISC 2000. Lect. Notes Comput. Sci, 2015 (2001), 64-72.

[18] Canfield E. R., Gao Z., Greenhill C., McKay B. D., Robinson R. W., "Asymptotic enumeration of correlation-immune Boolean functions", Cryptography and Commun., 2:1 (2010), 111-126.

[19] Chor B., Goldreich O., Hastad J., Friedman J., Rudich S., Smolensky R., "The bit extraction problem or t-resilent functions", IEEE Symp. Found. Comput. Sci., 26 (1985), 396-407.

[20] Gopalakrishnan K., Stinson D. R., "Three characterizations of non-binary correlationimmune and resilient functions", Des, Codes and Cryptogr., 5 (1995), 241-251.

[21] Siegenthaler, T., "Correlation-immunity of nonlinear combining functions for cryptographic applications", IEEE Trans. Inf. Theory, 30:5 (1984), 776-780.

[22] Stinson D., Massey J., "An infinite class of counterexamples to a conjecture concerning non-linear resilient functions", J. Cryptology, 8:3 (1995), 167-173.

[23] Zhang X., Zheng Y., "Cryptographically resilient functions", IEEE Trans. Inf. Theory, 43:5 (1997), 1740-1747. 